\title{
Optogenetic Analysis of Allosteric Control in Protein Tyrosine Phosphatases
}

\author{
Akarawin Hongdusit ${ }^{\mathrm{a}}$ and Jerome M. Fox ${ }^{\mathrm{a}^{*}}$ \\ aDepartment of Chemical and Biological Engineering, University of Colorado Boulder, \\ 3415 Colorado Avenue, Boulder, CO, 80303 \\ *To whom correspondence should be addressed. E-mail: jerome.fox@colorado.edu
}

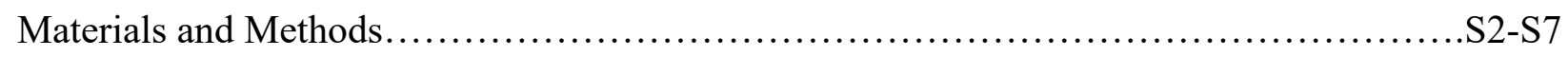

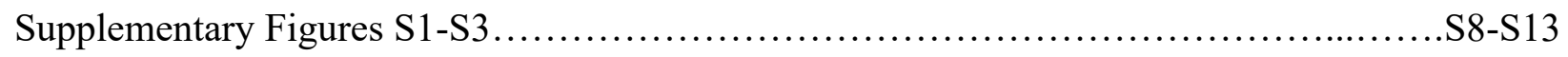

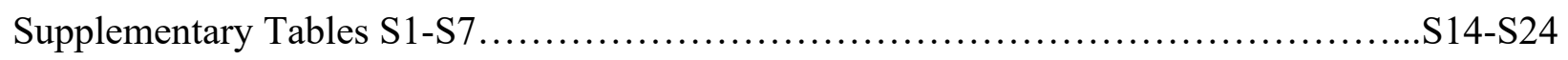




\section{Materials and Methods}

Materials. We purchased competent Turbo and BL21(DE3) E.coli cells from New England Biolabs (Ipswich, MA); BBR from Cayman Chemical (Ann Arbor, MI); tyrosine phosphopeptide (RRLIEDAEpYAARG), p-nitrophynyl, 4-methylumbelliferyl phosphate, and a Malachite Green assay kit from MilliporeSigma (St. Louis, MO); the EGFR-derived phosphopeptide (DADEpYLIPQQG) from R\&D Systems (Minneapolis, MN); and all other reagents from MilliporeSigma or Thermo Fisher. We purified PTP1B $1_{1-321}$ (UniProt P18031) and TCPTP ${ }_{1-317}$ (UniProt P17706) and fusions containing both (i) LOV2 (UniProt O49003) and (ii) either modified or unmodified versions of each PTP as described below.

Molecular cloning. We used a C-terminal polyhistidine tag (LEHHHHHH) for all constructs examined in this study. For all chimeras, this tag is attached to the J $\alpha$ helix of LOV2; for all isolated PTPs, it is attached to a disordered C-terminal region (i.e., an unstructured region located beyond the $\alpha 7$ helix). The tag's identical position on different constructs makes it unlikely to contribute to differences in activity between those constructs.

We designed TCPTP-LOV2 chimeras by aligning the catalytic domains of PTP1B (residues 1-298) and TCPTP (residues 1-296) and selecting identical residues as fusion points (Fig. 2B). We amplified segments of TCPTP from PBG100-TCPTP (Addgene, 33365) and segments of PTP1B-LOV2 from a plasmid containing the full-length chimera ${ }^{1}$. For TCPTP (residues 1-281 and 1-317) and chimeras 1-8, we assembled DNA fragments with overlap extension PCR and inserted them into a $\mathrm{pET} 16 \mathrm{~b}$ vector at BsmBI/BamHI restriction sites. For chimera 9, we assembled the final DNA fragment and placed it directly into the pET16b vector with Gibson assembly $\left(50^{\circ} \mathrm{C}\right.$ for $\left.1 \mathrm{hr}\right)$. Finally, we amplified PTP1B (residues 1-321) from a 
plasmid encoding the full catalytic domain (a kind gift from the Tonks group of Cold Spring Harbor Laboratory) and inserted it into a pET16b vector at NcoI/BamHI restriction sites.

We mutated PTP1B by using mutagenic primers (Table S4) to amplify the parent plasmid, and we mutated TCPTP and TCPTP $v$ by using mutagenic overhangs (Table S3). We integrated mutated constructs into plasmids by using restriction digest (PTP1B) or Gibson assembly (TCPTP) as above. We confirmed the sequences of all chimeras with Sanger sequencing (QuintaraBio).

Protein Expression and Purification. We overexpressed all proteins in E.coli by carrying out the following steps: (i) We transformed E.coli BL21(DE3) cells (New England Biolabs) with each plasmid. (ii) We used one colony from each plate to inoculate $20 \mathrm{~mL}$ of lysogeny broth (LB) media supplemented with $100 \mathrm{mg} / \mathrm{L}$ carbenicillin, and we incubated these cultures in a shaker at $37^{\circ} \mathrm{C}$ for at least $5 \mathrm{hr}$. (iii) We used each $20-\mathrm{mL}$ culture to inoculate $1 \mathrm{~L}$ of rich induction media (20 g/L tryptone, $10 \mathrm{~g} / \mathrm{L}$ yeast extract, $5 \mathrm{~g} / \mathrm{L} \mathrm{NaCl}, 4 \mathrm{~g} / \mathrm{L}$ M9 slats, $4 \mathrm{~g} / \mathrm{L}$ glucose, and $100 \mathrm{mg} / \mathrm{L}$ carbenicillin), which we incubated in a shaker at $37^{\circ} \mathrm{C}$ until the $\mathrm{OD}_{600}$ reached 0.6. (iv) We induced protein expression by adding $500 \mu \mathrm{L}$ of $1 \mathrm{M}$ solution of isopropyl b-D-1-thiogalactopyranoside (IPTG) to each culture (with this dilution, [IPTG] $=1 \mathrm{mM}$ ) and lowered the temperature of the shaker to $22^{\circ} \mathrm{C}$. (v) We pelleted the cells at $20 \mathrm{~h}$ after induction (3950 xg, 20 min; JA-10 Beckman Coulter).

We purified all proteins by using fast protein liquid chromatography (FPLC) using the following steps: (i) We lysed the cells by adding the following components to each gram of pellet: $4 \mathrm{~mL}$ of B-PER (Thermo Fisher Scientific, Inc.), 1 mg MgSO4, 2 mg Na-p-Tosyl-Larginine methyl ester hydrochloride, $1.25 \mathrm{mg}$ tris(2-carboxyethyl)phosphine (TCEP), $3.75 \mu \mathrm{l}$ 
phenylmethylsulfonyl fluoride, $1 \mathrm{mg}$ Lysozyme, and $10 \mu \mathrm{l}$ DNase. We mixed this suspension to homogeneity and rocked the mixture for $1 \mathrm{~h}$ at room temperature $\left(22^{\circ} \mathrm{C}\right)$. (ii) We pelleted the cell debris (3950 xg, $60 \mathrm{~min}$ ) and collected the supernatant. (iii) We added saturated ammonium sulfate solution to the supernatant at $10 \% \mathrm{v} / \mathrm{v}$ and immediately pelleted the mixture $(3950 \mathrm{xg}, 60$ min). (iv) We collected the supernatant and ran the solution through $0.22 \mu \mathrm{m}$ filter. (v) We exchanged the supernatant into a Tris-HCl buffer ( $50 \mathrm{mM}$ Tris-HCl, $0.5 \mathrm{mM}$ TCEP, $\mathrm{pH}$ 7.5), ran the exchanged solution over a Ni column (HisTrap HP [Ni], GE Healthcare), and eluted the protein with a $0-100 \%$ imidazole gradient $(50 \mathrm{mM}$ Tris- $\mathrm{HCl}, 0.5 \mathrm{mM}$ TCEP, $500 \mathrm{mM}$ imidazole, pH 7.5). (vi) We exchanged the protein solution into HEPES buffer (50 mM HEPES, $0.5 \mathrm{mM}$ TCEP, pH 7.5), ran the solution over an anion exchange column (HiPrep Q HP 16/10, GE Healthcare), and eluted the protein with a $0-100 \%$ salt gradient (50 mM HEPES, $0.5 \mathrm{mM}$ TCEP, $500 \mathrm{mM} \mathrm{NaCl}, \mathrm{pH}$ 7.5). We confirmed the purity of all protein preparations with SDS-PAGE, and we stored each protein at $-80^{\circ} \mathrm{C}(50 \mathrm{mM}$ HEPES, $0.5 \mathrm{mM}$ TCEP, $20 \mathrm{v} / \mathrm{v} \%$ glycerol, $\mathrm{pH} 7.5)$

Analysis of photoswitching. We characterized the photoswitchability of various PTP-LOV2 chimeras as described previously ${ }^{1}$. Briefly, we used two clear 96-well plates (\#3370, Costar) to prepare identical $100-\mu \mathrm{L}$ reactions containing buffer (50 mM HEPES, $0.5 \mathrm{mM}$ Tris(2carboxyethyl)phosphine hydrochloride, $\mathrm{pH} 7.5)$, substrate (500 $\mu \mathrm{M}$ 4-methylumbelliferyl phosphate [4-MUP]), and enzyme (5 nM). After adding enzyme, we placed the first 96-well plate in the dark and the second plate in a blue light chamber (We obtained the blue light from Mightex Systems [SLS-0301-C, $455 \mathrm{~nm}$ ]; Figure S2). We carried out all reactions at room temperature $\left(22^{\circ} \mathrm{C}\right)$. At $7,14,21,28,35$ and 42 minutes, we measured the formation of 4methylumbelliferone $\left(\lambda_{\mathrm{ex}} / \lambda_{\mathrm{em}}=365 \mathrm{~nm} / 450 \mathrm{~nm}\right)$ with a SpectraMax M2 plate reader. We note: 
The fluorescent 4-MUP substrate, which enhances the sensitivity of our assay, allows us to measure hydrolysis rates over extended periods of time while remaining in the initial rate regime. Neither the light used to measure product release nor the time ( $\sim 20-30$ seconds) required to move plates to and from the plate reader had a detectable influence on PTP activity.

We characterized the most photoswitchable chimera by measuring PTP-catalyzed hydrolysis of pNPP in the presence and absence of blue light (Fig. 2D). Briefly, we prepared $100-\mu \mathrm{L}$ reactions consisting of buffer (50 mM HEPES, $0.5 \mathrm{mM}$ TCEP, $\mathrm{pH} 7.5)$, substrate $(0.2$, $0.5,1.52 .5,5,10$ and $15 \mathrm{mM} \mathrm{pNPP})$, and enzyme ( $25 \mathrm{nM})$; at 4, 8, 12, 16 and 20 minutes after initiating the reaction, we measured the production of p-nitrophenol ( $405_{\mathrm{abs}}$ ) on a SpectraMax M2 plate reader; and we used DataGraph to fit initial rates to a Michaelis-Menten model of enzyme kinetics. Final values of $k_{c a t}$ and $K_{m}$ reflect the mean of independent estimates determined from three Michaelis-Menten curves; error in $k_{c a t}$ and $K_{m}$ reflects the standard error of those estimates.

Enzyme kinetics. We measured the activities of PTPs on various substrates by using initial rate measurements to construct Michaelis-Menten curves (Figures S3). Each reaction consisted of buffer (50 mM HEPES, $0.5 \mathrm{mM}$ TCEP, $\mathrm{pH} 7.5)$, enzyme ( $25 \mathrm{nM})$, and the following substrate concentrations: $\operatorname{pNPP}(0.2,0.5,1.52 .5,5,10,15$ and $20 \mathrm{mM})$; 4-MUP $(25,50,150,250,500$, 1000, 1500 and $2000 \mu \mathrm{M})$; and RRLIEDAEpYAARG or DADEpYLIPQQG $(5,15,25,50,100$, 150, 200, $250 \mu \mathrm{M}$ peptide). For pNPP and 4-MUP, we monitored the production of 4-nitrophenyl ( $\left.405_{\mathrm{abs}}\right)$ or $4-\mathrm{MU}\left(365_{\mathrm{ex}}\right.$ and $\left.450_{\mathrm{em}}\right)$, respectively, at 1-minute intervals for 6 minutes. For the peptides, we used the Malachite Green assay kit (MAK307, MilliporeSigma) to measure phosphate release. In brief, we added $10 \mu \mathrm{L}$ of malachite green working solution (included in the 
kit) to $40-\mu \mathrm{L}$ reactions at 2, 4, 6 minutes after initiating the reactions, and we measured the absorbance at $620 \mathrm{~nm}$ after 30 minutes of incubation.

We compared the susceptibilities of TCPTP and TC-PTP ${ }_{\mathrm{V} 9}$ to inhibition by BBR by supplementing the pNPP reactions $(0.5,1.5,2.5,5,10$, and $15 \mathrm{mM})$ with $0,5,10,20 \mu \mathrm{M}$ of BBR (0.025-0.1\% DMSO). Here, we monitored the production of 4-nitrophenyl (405 abs) at 30 seconds intervals for 6 minutes. We used MATLAB's "nlinfit" and "fminsearch" functions to fit initialrate measurements to either a Michaelis-Menten model (no inhibitor) or each of four models of inhibition (with inhibitor; here, we chose competitive, noncompetitive, uncompetitive, and mixed inhibition $^{2}$ ). (iii) We used an F- test to compare the fits of the mixed model to the singleparameter model with the lowest sum of squared errors; the noncompetitive model was the best model in each case. (iv) We estimated $\mathrm{IC}_{50}$ 's by using the noncompetitive model to determine the inhibitor concentration required to reduce initial rates by $50 \%$ on $15 \mathrm{mM}$ pNPP. We used the MATLAB function "nlparci" to determine the confidence intervals of kinetic parameters and propagated those intervals to estimate the corresponding confidence on IC50's.

X-ray crystallography. We prepared crystals of PTP1B using hanging drop vapor diffusion. In brief, we prepared $8-\mu \mathrm{L}$ droplets of protein solution on plastic supports by combining $6 \mu \mathrm{L}$ of crystallization solution (100 mM HEPES, $200 \mathrm{mM}$ magnesium acetate, and 14\% polyethylene glycol 8000, pH 7.5) with $2 \mu \mathrm{L}$ of PTP1B ( 600 $\mu \mathrm{M}$ PTP1B, $50 \mathrm{mM}$ HEPES, pH 7.3), and we inverted these supports over reservoirs containing $1 \mathrm{ml}$ of crystallization solution for one week at $4^{\circ} \mathrm{C}$ (EasyXtal CrystalSupport, Qiagen). We prepared all crystals for freezing by soaking them in cryoprotectant formed by combining a crystallization solution (100 mM HEPES, $200 \mathrm{mM}$ magnesium acetate, and 25\% polyethylene glycol $8000, \mathrm{pH} 7.5$ ) with glycerol in a 7:3 mixture. 
We collected X-ray diffraction data through the Collaborative Crystallography Program at Lawrence Berkeley National Lab (ALS ENABLE, beamline 5.2.1, $100 \mathrm{~K}, 0.97741 \AA$ ). We performed integration, scaling, and merging of X-ray diffraction data using the xia2 software package $^{3}$, and we carried out molecular replacement and structure refinement with the PHENIX graphical interface ${ }^{4}$, supplemented with manual model adjustment in $\mathrm{COOT}^{5}$ and one round of refinement with PDB-REDO ${ }^{6}$. Our model of PTP1B (PDB entry 6b90) included both open and closed conformations of the WPD loop and, thus, allowed us to assign occupancies to those conformations during refinement. Table S7 reports the statistics for X-ray crystallography. 


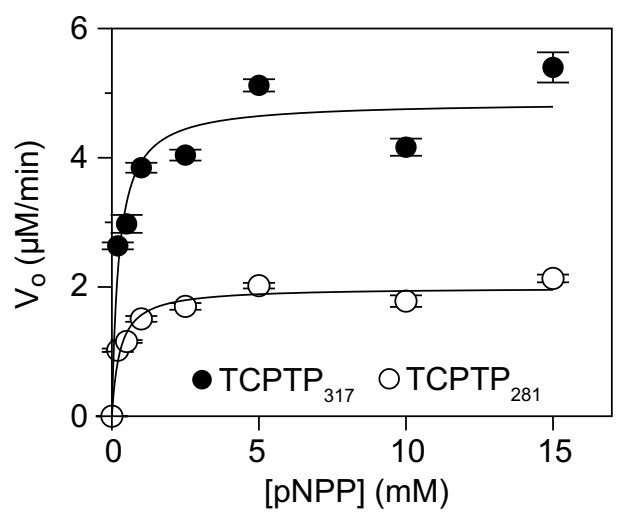

Figure S1. Saturation curves of $\mathrm{TCPTP}_{317}$ and $\mathrm{TCPTP}_{281}$. Initial rates of $\mathrm{pNPP}$ hydrolysis by TCPTP $_{317}$ (black) and TC-PTP 281 (white). Lines denote fits to a Michalis-Menten model of enzyme kinetics. The plotted data depict the mean and SE of $\mathrm{n}=3$ independent reactions. Kinetic parameters appear in Table S5. 


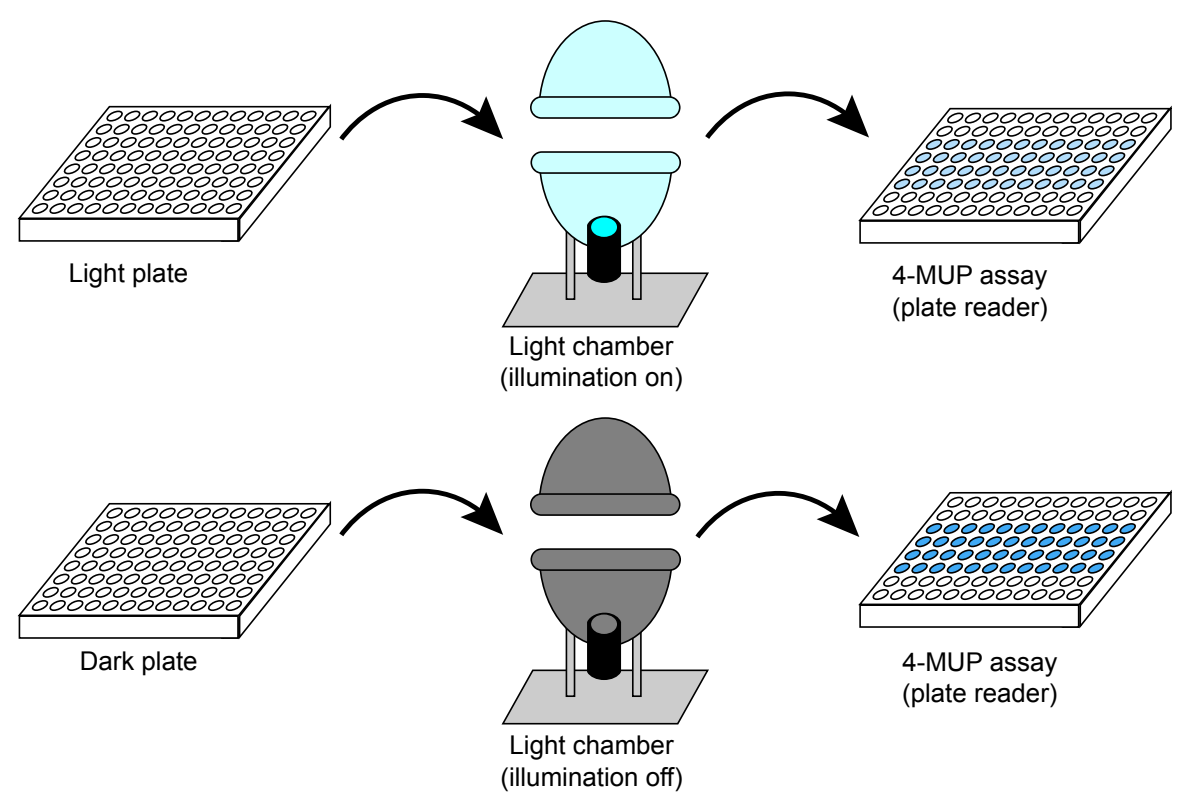

Figure S2. Schematic of photoswitching assay. We prepared two identical 96-well plates with buffer, substrate, and enzyme - hereafter, referred to as the dark plate and the light plate. We placed the light plate in a light chamber equipped with a blue light, which was turned on; we placed the dark plate in a similar chamber with no illumination. At discrete time points, we removed the plates and monitored the hydrolysis of 4-methylumbelliferyl phosphate by using a plate reader to measure the formation of 4-methylumbellifone $\left(\lambda_{\mathrm{ex}} / \lambda_{\mathrm{em}}=365 \mathrm{~nm} / 450 \mathrm{~nm}\right)$. Details of light chamber assembly (including photographs) appear in our prior study ${ }^{1}$ 

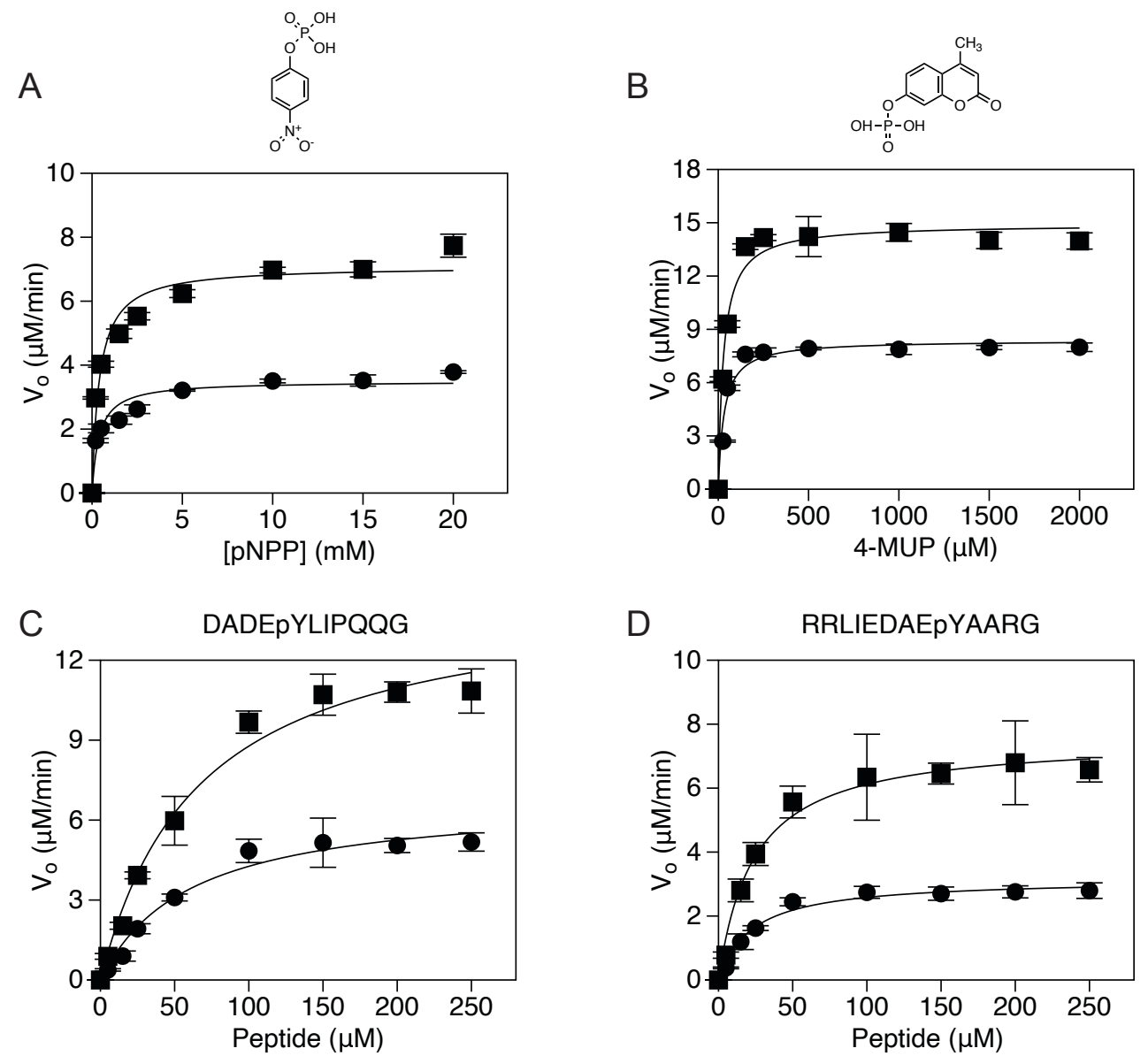

Figure S3. Comparison of the activities of TCPTP and TCPTPvg on different substrates. (A-D) Initial rates of dephosphorylation of (A) pNPP, (B) 4-MUP, (C) E-PEP and (D) P-PEP by TCPTP (circle) and TCPTP $v$ (square). Lines denote fits to the Michaelis-Menten model of enzyme kinetics. The plotted data depict the mean and SE of $\mathrm{n}=3$ independent reactions Kinetic parameters appear in Table S5. 

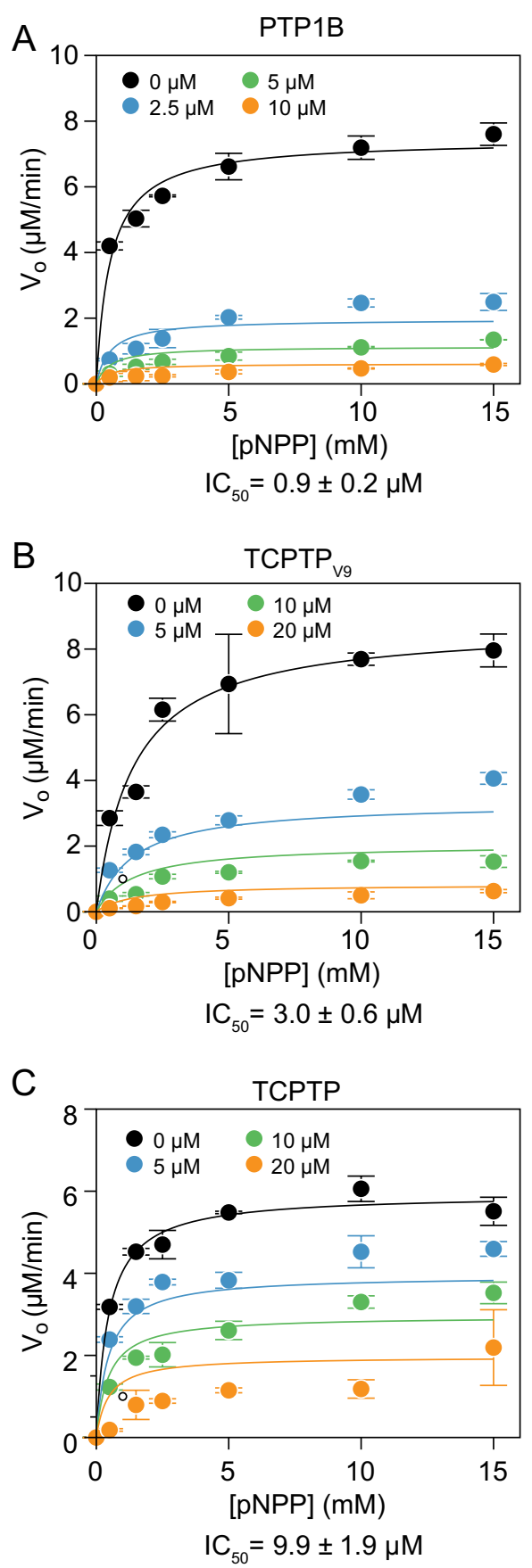

Figure S4. Inhibition of variants of TCPTP by BBR. (A-C) Initial rates of pNPP hydrolysis by (A) PTP1B, (B) TCPTP $v 9$ and (C) TCPTP in the presence of increasing concentrations of BBR. Lines denote models to models for noncompetitive inhibition (i.e., the best-fit models). The plotted data depict the mean and SE of $\mathrm{n}=3$ independent reactions. Inhibition parameters and kinetic constants appear in Tables S5 and S6. 
A

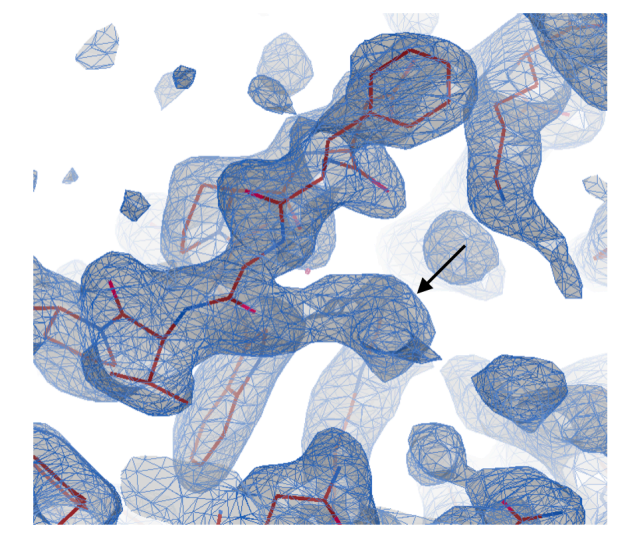

B

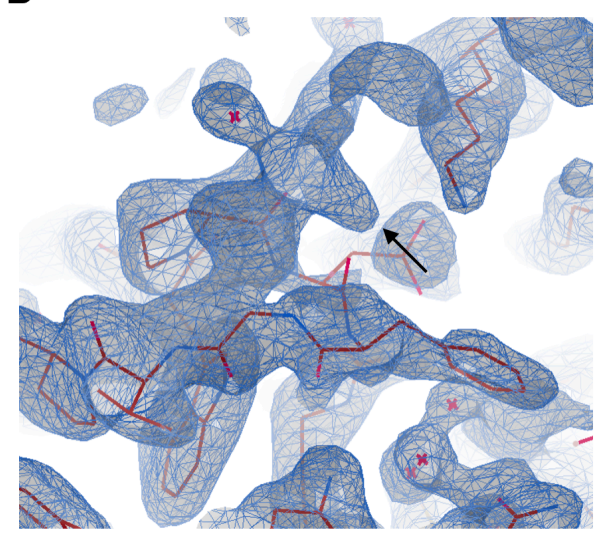

Figure S5. Single-conformer models of PTP1B (D289A). Crystal structures of PTP1B (D289A) solved by modeling the WPD loop in either (A) an open conformation or (B) a closed conformation (model PDB entries $3 \mathrm{a} 5 \mathrm{j}$ and $1 \mathrm{sug}$, respectively). In each case, the $0.80 \sigma 2 \mathrm{Fo}-\mathrm{Fc}$ electron density supports the modeled conformation, but additional density aligns with the unmodeled conformation (arrows). This secondary density is strongest for the closed conformation, an indication that the open conformation has the highest occupancy. The unexplained density in the single-conformer models supports the use of a multi-conformer model (i.e., PTP1B with both open and closed WPD loops) for refinement. 


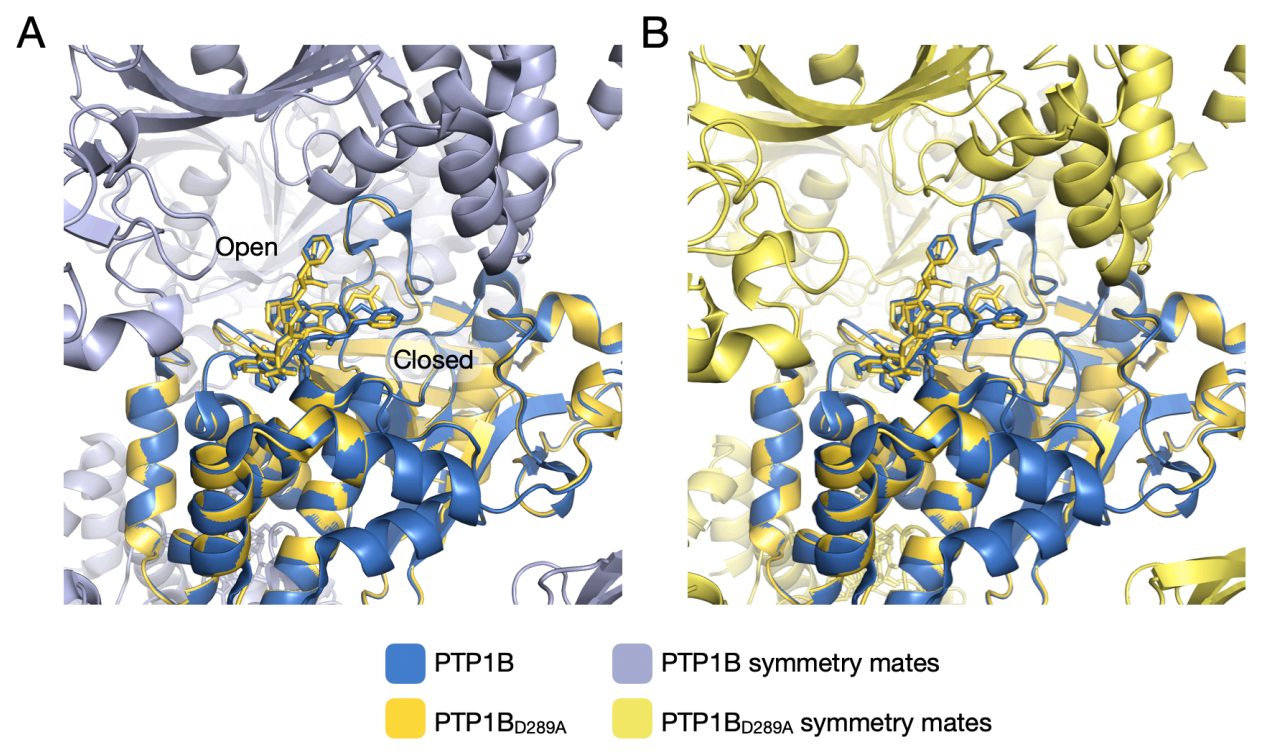

Figure S6. Analysis of symmetry mates within PTP1B crystals. Aligned crystal structures of (A) PTP1B and (B) PTP1B (D289A) with symmetry mates located within $12 \AA$ (PDB entries 6b8x and 7ken, respectively). These crystals have the same space group (P 3121) and a nearly identical unit cell $(\mathrm{a}=\mathrm{b}=88.3-89.5, \mathrm{c}=104-106, \alpha=\beta=90, \mathrm{\gamma}=120)$. The WPD loop (sticks) is not located near a major crystal contact. 
Table S1. Amino acid sequences of heterologously expressed proteins

\#

PS1 1-288:289-296:297-438:439446

PS2

PS3

PS4

PS5
$1-280: 281-296: 297-438: 439$ 446

1-285:286-296:297-438:439446

$1-278: 279-296: 297-438: 439-$ 446

1-272:273-296:297-438:439446
Sequence

MPTTIEREFEELDTQRRWQPLYLEIRNESHDYPHRVAKFPENRNRN RYRDVSPYDHSRVKLQNAENDYINASLVDIEEAQRSYILTQGPLPN TCCHFWLMVWQQKTKAVVMLNRIVEKESVKCAQYWPTDDQEM LFKETGFSVKLLSEDVKSYYTVHLLQLENINSGETRTISHFHYTTW PDFGVPESPASFLNFLFKVRESGSLNPDHGPAVIHCSAGIGRSGTFS LVDTCLVLMEKGDDINIKQVLLNMRKYRMGLIQTPDQLRFSYMAI IEGAKCIKGDSSIQKRWKELSHEDAATLERIEKNFVITDPRLPDNPII FASDSFLQLTEYSREEILGRNCRFLQGPETDRATVRKIRDAIDNQTE VTVQLINYTKSGKKFWNLFHLQPMRDQKGDVQYFIGVQLDGTEH VRDAAEREGVMLIKKTAENIDEAAKELLLEHHHHHH

MPTTIEREFEELDTQRRWQPLYLEIRNESHDYPHRVAKFPENRNRN RYRDVSPYDHSRVKLQNAENDYINASLVDIEEAQRSYILTQGPLPN TCCHFWLMVWQQKTKAVVMLNRIVEKESVKCAQYWPTDDQEM LFKETGFSVKLLSEDVKSYYTVHLLQLENINSGETRTISHFHYTTW PDFGVPESPASFLNFLFKVRESGSLNPDHGPAVIHCSAGIGRSGTFS LVDTCLVLMEKGDDINIKQVLLNMRKYRMGLIQTPDQLRFSYMAI IEGAKCIKGDSSIQDQWKELSHEDAATLERIEKNFVITDPRLPDNPII FASDSFLQLTEYSREEILGRNCRFLQGPETDRATVRKIRDAIDNQTE VTVQLINYTKSGKKFWNLFHLQPMRDQKGDVQYFIGVQLDGTEH VRDAAEREGVMLIKKTAENIDEAAKELLEHHHHHH

MPTTIEREFEELDTQRRWQPLYLEIRNESHDYPHRVAKFPENRNRN RYRDVSPYDHSRVKLQNAENDYINASLVDIEEAQRSYILTQGPLPN TCCHFWLMVWQQKTKAVVMLNRIVEKESVKCAQYWPTDDQEM LFKETGFSVKLLSEDVKSYYTVHLLQLENINSGETRTISHFHYTTW PDFGVPESPASFLNFLFKVRESGSLNPDHGPAVIHCSAGIGRSGTFS LVDTCLVLMEKGDDINIKQVLLNMRKYRMGLIQTPDQLRFSYMAI IEGAKCIKGDSSVQDQWKELSHEDAATLERIEKNFVITDPRLPDNPI IFASDSFLQLTEYSREEILGRNCRFLQGPETDRATVRKIRDAIDNQT EVTVQLINYTKSGKKFWNLFHLQPMRDQKGDVQYFIGVQLDGTE HVRDAAEREGVMLIKKTAENIDEAAKELLEHHHHHH

MPTTIEREFEELDTQRRWQPLYLEIRNESHDYPHRVAKFPENRNRN RYRDVSPYDHSRVKLQNAENDYINASLVDIEEAQRSYILTQGPLPN TCCHFWLMVWQQKTKAVVMLNRIVEKESVKCAQYWPTDDQEM LFKETGFSVKLLSEDVKSYYTVHLLQLENINSGETRTISHFHYTTW PDFGVPESPASFLNFLFKVRESGSLNPDHGPAVIHCSAGIGRSGTFS LVDTCLVLMEKGDDINIKQVLLNMRKYRMGLIQTPDQLRFSYMAI IEGAKCIMGDSSVQDQWKELSHEDAATLERIEKNFVITDPRLPDNPI IFASDSFLQLTEYSREEILGRNCRFLQGPETDRATVRKIRDAIDNQT EVTVQLINYTKSGKKFWNLFHLQPMRDQKGDVQYFIGVQLDGTE HVRDAAEREGVMLIKKTAENIDEAAKELLEHHHHHH

MPTTIEREFEELDTQRRWQPLYLEIRNESHDYPHRVAKFPENRNRN RYRDVSPYDHSRVKLQNAENDYINASLVDIEEAQRSYILTQGPLPN TCCHFWLMVWQQKTKAVVMLNRIVEKESVKCAQYWPTDDQEM LFKETGFSVKLLSEDVKSYYTVHLLQLENINSGETRTISHFHYTTW PDFGVPESPASFLNFLFKVRESGSLNPDHGPAVIHCSAGIGRSGTFS LVDTCLVLMEKGDDINIKQVLLNMRKYRMGLIQTPDQLRFSYMAI IEGAKFIMGDSSVQDQWKELSHEDAATLERIEKNFVITDPRLPDNPI IFASDSFLQLTEYSREEILGRNCRFLQGPETDRATVRKIRDAIDNQT EVTVQLINYTKSGKKFWNLFHLQPMRDQKGDVQYFIGVQLDGTE HVRDAAEREGVMLIKKTAENIDEAAKELLEHHHHHH 
PS6

1-270:271-296:297-438:439446

PS7

1-262:263-296:297-438:439446

PS8

1-233:234-299:297-441:442449

PS9

1-114:115-121:122-233:234299:297-441:442-449

TCPTP 1-317:318-325

TCPTP

$1-281: 282-289$
MPTTIEREFEELDTQRRWQPLYLEIRNESHDYPHRVAKFPENRNRN RYRDVSPYDHSRVKLQNAENDYINASLVDIEEAQRSYILTQGPLPN TCCHFWLMVWQQKTKAVVMLNRIVEKESVKCAQYWPTDDQEM LFKETGFSVKLLSEDVKSYYTVHLLQLENINSGETRTISHFHYTTW PDFGVPESPASFLNFLFKVRESGSLNPDHGPAVIHCSAGIGRSGTFS LVDTCLVLMEKGDDINIKQVLLNMRKYRMGLIQTPDQLRFSYM AVIEGAKFIMGDSSVQDQWKELSHEDAATLERIEKNFVITDPRLPD NPIIFASDSFLQLTEYSREEILGRNCRFLQGPETDRATVRKIRDAIDN QTEVTVQLINYTKSGKKFWNLFHLQPMRDQKGDVQYFIGVQLDG TEHVRDAAEREGVMLIKKTAENIDEAAKELLEHHHHHH

MPTTIEREFEELDTQRRWQPLYLEIRNESHDYPHRVAKFPENRNRN RYRDVSPYDHSRVKLQNAENDYINASLVDIEEAQRSYILTQGPLPN TCCHFWLMVWQQKTKAVVMLNRIVEKESVKCAQYWPTDDQEM LFKETGFSVKLLSEDVKSYYTVHLLQLENINSGETRTISHFHYTTW PDFGVPESPASFLNFLFKVRESGSLNPDHGPAVIHCSAGIGRSGTFS LVDTCLVLMEKGDDINIKQVLLNMRKYRMGLIQTPDQLRFSYLAV IEGAKFIMGDSSVQDQWKELSHEDAATLERIEKNFVITDPRLPD NPIIFASDSFLQLTEYSREEILGRNCRFLQGPETDRATVRKIRDAIDN QTEVTVQLINYTKSGKKFWNLFHLQPMRDQKGDVQYFIGVQLDG TEHVRDAAEREGVMLIKKTAENIDEAAKELLEHHHHHH

MPTTIEREFEELDTQRRWQPLYLEIRNESHDYPHRVAKFPENRNRN RYRDVSPYDHSRVKLQNAENDYINASLVDIEEAQRSYILTQGPLPN TCCHFWLMVWQQKTKAVVMLNRIVEKESVKCAQYWPTDDQEM LFKETGFSVKLLSEDVKSYYTVHLLQLENINSGETRTISHFHYTTW PDFGVPESPASFLNFLFKVRESGSLNPDHGPAVIHCSAGIGRSGTFS LVDTCLLLMDKRKDPSSVDIKKVLLEMRKFRMGLIQTADQLRFSY LAVIEGAKFIMGDSSVQDQWKELSHEDAATLERIEKNFVITDPRLP DNPIIFASDSFLQLTEYSREEILGRNCRFLQGPETDRATVRKIRDAID NQTEVTVQLINYTKSGKKFWNLFHLQPMRDQKGDVQYFIGVQLD GTEHVRDAAEREGVMLIKKTAENIDEAAKELLEHHHHHH

MPTTIEREFEELDTQRRWQPLYLEIRNESHDYPHRVAKFPENRNRN RYRDVSPYDHSRVKLQNAENDYINASLVDIEEAQRSYILTQGPLPN TCCHFWLMVWQQKTKAVVMLNRVMEKGSLKCAQYWPTDDQE MLFKETGFSVKLLSEDVKSYYTVHLLQLENINSGETRTISHFHYTT WPDFGVPESPASFLNFLFKVRESGSLNPDHGPAVIHCSAGIGRSGTF SLVDTCLLLMDKRKDPSSVDIKKVLLEMRKFRMGLIQTADQLRFS YLAVIEGAKFIMGDSSVQDQWKELSHEDAATLERIEKNFVITDPRL PDNPIIFASDSFLQLTEYSREEILGRNCRFLQGPETDRATVRKIRDAI DNQTEVTVQLINYTKSGKKFWNLFHLQPMRDQKGDVQYFIGVQL DGTEHVRDAAEREGVMLIKKTAENIDEAAKELLEHHHHHH MPTTIEREFEELDTQRRWQPLYLEIRNESHDYPHRVAKFPENRNRN RYRDVSPYDHSRVKLQNAENDYINASLVDIEEAQRSYILTQGPLPN TCCHFWLMVWQQKTKAVVMLNRIVEKESVKCAQYWPTDDQEM LFKETGFSVKLLSEDVKSYYTVHLLQLENINSGETRTISHFHYTTW PDFGVPESPASFLNFLFKVRESGSLNPDHGPAVIHCSAGIGRSGTFS LVDTCLVLMEKGDDINIKQVLLNMRKYRMGLIQTPDQLRFSYMAI IEGAKCIKGDSSIQKRWKELSKEDLSPAFDHSPNKIMTEKYNGNRL EHHHHHH

MPTTIEREFEELDTQRRWQPLYLEIRNESHDYPHRVAKFPENRNRN RYRDVSPYDHSRVKLQNAENDYINASLVDIEEAQRSYILTQGPLPN TCCHFWLMVWQQKTKAVVMLNRIVEKESVKCAQYWPTDDQEM LFKETGFSVKLLSEDVKSYYTVHLLQLENINSGETRTISHFHYTTW PDFGVPESPASFLNFLFKVRESGSLNPDHGPAVIHCSAGIGRSGTFS LVDTCLVLMEKGDDINIKQVLLNMRKYRMGLIQTPDQLRFSYMAI IEGAKCILEHHHHHH 
1-114:115-121:122-233:234-

299:300-320:331-338

$1-317: 318-325$

K287A

ТСРТP

V9-290A

PTP1B

$1-321: 322-329$

PTP1B

S286A

PTP1B
D289A

$P T P 1 B$
$D 289 A$

$1-321: 322-329$

1-114:115-121:122-233:234299:300-320:331-338

\section{1-321:322-329}

PTP1B

E293A
MPTTIEREFEELDTQRRWQPLYLEIRNESHDYPHRVAKFPENRNRN RYRDVSPYDHSRVKLQNAENDYINASLVDIEEAQRSYILTQGPLPN TCCHFWLMVWQQKTKAVVMLNRVMEKGSLKCAQYWPTDDQE MLFKETGFSVKLLSEDVKSYYTVHLLQLENINSGETRTISHFHYTT WPDFGVPESPASFLNFLFKVRESGSLNPDHGPAVIHCSAGIGRSGTF SLVDTCLLLMDKRKDPSSVDIKKVLLEMRKFRMGLIQTADQLRFS YLAVIEGAKFIMGDSSVQDQWKELSHEDLSPAFDHSPNKIMTEKY NGNRLEHHHHHH

MPTTIEREFEELDTQRRWQPLYLEIRNESHDYPHRVAKFPENRNRN RYRDVSPYDHSRVKLQNAENDYINASLVDIEEAQRSYILTQGPLPN TCCHFWLMVWQQKTKAVVMLNRIVEKESVKCAQYWPTDDQEM LFKETGFSVKLLSEDVKSYYTVHLLQLENINSGETRTISHFHYTTW PDFGVPESPASFLNFLFKVRESGSLNPDHGPAVIHCSAGIGRSGTFS LVDTCLVLMEKGDDINIKQVLLNMRKYRMGLIQTPDQLRFSYMAI IEGAKCIKGDSSIQARWKELSKEDLSPAFDHSPNKIMTEKYNGNRL EHHHHHH

MPTTIEREFEELDTQRRWQPLYLEIRNESHDYPHRVAKFPENRNRN RYRDVSPYDHSRVKLQNAENDYINASLVDIEEAQRSYILTQGPLPN TCCHFWLMVWQQKTKAVVMLNRVMEKGSLKCAQYWPTDDQE MLFKETGFSVKLLSEDVKSYYTVHLLQLENINSGETRTISHFHYTT WPDFGVPESPASFLNFLFKVRESGSLNPDHGPAVIHCSAGIGRSGTF SLVDTCLLLMDKRKDPSSVDIKKVLLEMRKFRMGLIQTADQLRFS YLAVIEGAKFIMGDSSVQAQWKELSHEDLSPAFDHSPNKIMTEKY NGNRLEHHHHHH

MEMEKEFEQIDKSGSWAAIYQDIRHEASDFPCRVAKLPKNKNRNR YRDVSPFDHSRIKLHQEDNDYINASLIKMEEAQRSYILTQGPLPNTC GHFWEMVWEQKSRGVVMLNRVMEKGSLKCAQYWPQKEEKEMI FEDTNLKLTLISEDIKSYYTVRQLELENLTTQETREILHFHYTTWPD FGVPESPASFLNFLFKVRESGSLSPEHGPVVVHCSAGIGRSGTFCLA DTCLLLMDKRKDPSSVDIKKVLLEMRKFRMGLIQTADQLRFSYLA VIEGAKFIMGDSSVQDQWKELSHEDLEPPPEHIPPPPRPPKRILEPH NLEHHHHHH

MEMEKEFEQIDKSGSWAAIYQDIRHEASDFPCRVAKLPKNKNRNR YRDVSPFDHSRIKLHQEDNDYINASLIKMEEAQRSYILTQGPLPNTC GHFWEMVWEQKSRGVVMLNRVMEKGSLKCAQYWPQKEEKEMI FEDTNLKLTLISEDIKSYYTVRQLELENLTTQETREILHFHYTTWPD FGVPESPASFLNFLFKVRESGSLSPEHGPVVVHCSAGIGRSGTFCLA DTCLLLMDKRKDPSSVDIKKVLLEMRKFRMGLIQTADQLRFSYLA VIEGAKFIMGDSAVQDQWKELSHEDLEPPPEHIPPPPRPPKRILEPH NLEHHHHHH

MEMEKEFEQIDKSGSWAAIYQDIRHEASDFPCRVAKLPKNKNRNR YRDVSPFDHSRIKLHQEDNDYINASLIKMEEAQRSYILTQGPLPNTC GHFWEMVWEQKSRGVVMLNRVMEKGSLKCAQYWPQKEEKEMI FEDTNLKLTLISEDIKSYYTVRQLELENLTTQETREILHFHYTTWPD FGVPESPASFLNFLFKVRESGSLSPEHGPVVVHCSAGIGRSGTFCLA DTCLLLMDKRKDPSSVDIKKVLLEMRKFRMGLIQTADQLRFSYLA VIEGAKFIMGDSSVQAQWKELSHEDLEPPPEHIPPPPRPPKRILEPH NLEHHHHHH

MEMEKEFEQIDKSGSWAAIYQDIRHEASDFPCRVAKLPKNKNRNR YRDVSPFDHSRIKLHQEDNDYINASLIKMEEAQRSYILTQGPLPNTC GHFWEMVWEQKSRGVVMLNRVMEKGSLKCAQYWPQKEEKEMI FEDTNLKLTLISEDIKSYYTVRQLELENLTTQETREILHFHYTTWPD FGVPESPASFLNFLFKVRESGSLSPEHGPVVVHCSAGIGRSGTFCLA DTCLLLMDKRKDPSSVDIKKVLLEMRKFRMGLIQTADQLRFSYLA VIEGAKFIMGDSSVQDQWKALSHEDLEPPPEHIPPPPRPPKRILEPH NLEHHHHHH 


\begin{tabular}{r|ll}
$P T P 1 B$ & $1-321: 322-329$ & MEMEKEFEQIDKSGSWAAIYQDIRHEASDFPCRVAKLPKNKNRNR \\
E297A & YRDVSPFDHSRIKLHQEDNDYINASLIKMEEAQRSYILTQGPLPNTC \\
& GHFWEMVWEQKSRGVVMLNRVMEKGSLKCAQYWPQKEEKEMI \\
& FEDTNLKLTLISEDIKSYYTVRQLELENLTTQETREILHFHYTTWPD \\
& FGVPESPASFLNFLFKVRESGSLSPEHGPVVVHCSAGIGRSGTFCLA \\
& DTCLLLMDKRKDPSSDIKKVLLEMRKFRGLIQTADQLRFSLA \\
& VIEGAKFIMGDSSVQDQWKELSHADLEPPPEHIPPPPRPPKRILEPH \\
& NLEHHHHHH
\end{tabular}

${ }^{*}$ Residues are numbered according to the sequences of each chimera component: TCPTP (black), PTP1B (red), LOV2 (blue), and affinity tag (brown). 
Table S2. Primers used to assemble TCPTP-LOV2 chimeras.

\begin{tabular}{|c|c|c|c|c|}
\hline \# & ТСРTP & & PTP1B-L & 92 \\
\hline & F Primer & R Primer & F Primer & R Primer \\
\hline 1 & AAAAAGGTCTCC & GTAGGAGAAGCGCA & GAATGGGTCTTA & TTTTTGGATCCTCAG \\
\hline & CATGGGAATGCC & GCTGGTCTGGGGTCT & TTCAGACCCCAG & TGGTGGTGGTGGTG \\
\hline & CACCACCATCGA & GAATAAGACCCATT & ACCAGCTGCGCT & GTGCTCGAGAAGTT \\
\hline & $\mathrm{GC}$ & $\mathrm{C}$ & TCTCCTAC & $\begin{array}{l}\text { CTTTTGCCGCCTCAT } \\
\text { CAAT }\end{array}$ \\
\hline 2 & AAAAAGGTCTCC & GAATTTGGCACCTTC & CCCAGATCAACT & TTTTTGGATCCTCAG \\
\hline & CATGGGAATGCC & GATCACAGCCATGT & GAGATTCTCATA & TGGTGGTGGTGGTG \\
\hline & CACCACCATCGA & ATGAGAATCTCAGTT & CATGGCTGTGAT & GTGCTCGAGAAGTT \\
\hline & $\mathrm{GC}$ & GATCTGGG & CGAAGGTGCCA & CTTTTGCCGCCTCAT \\
\hline & & & AATTC & CAAT \\
\hline 3 & AAAAAGGTCTCC & CATGATGAATTTGGC & GATCAACTGAGA & TTTTTGGATCCTCAG \\
\hline & CATGGGAATGCC & ACCTTCGATTATAGC & TTCTCATACATG & TGGTGGTGGTGGTG \\
\hline & CACCACCATCGA & CATGTATGAGAATCT & GCTATAATCGAA & GTGCTCGAGAAGTT \\
\hline & $\mathrm{GC}$ & CAGTTGATC & GGTGCCAAATTC & CTTTTGCCGCCTCAT \\
\hline & & & ATCATG & CAAT \\
\hline 4 & AAAAAGGTCTCC & CACGGAAGAGTCCC & CATACATGGCTA & TTTTTGGATCCTCAG \\
\hline & CATGGGAATGCC & CCATGATACATTTTG & TAATAGAAGGA & TGGTGGTGGTGGTG \\
\hline & CACCACCATCGA & СТССТТСТАТТАТАG & GCAAAATGTATC & GTGCTCGAGAAGTT \\
\hline & & CCATGTATG & ATGGGGGACTCT & CTTTTGCCGCCTCAT \\
\hline & & & TCCGTG & CAAT \\
\hline 5 & AAAAAGGTCTCC & CTGCACGGAAGAGT & CATGGCTATAAT & TTTTTGGATCCTCAG \\
\hline & CATGGGAATGCC & СССССТТТАТАСАТТ & AGAAGGAGCAA & TGGTGGTGGTGGTG \\
\hline & CACCACCATCGA & TTGCТССТTCTATTA & AATGTATAAAGG & GTGCTCGAGAAGTT \\
\hline & $\mathrm{GC}$ & TAGCCATG & GGGACTCTTCCG & CTTTTGCCGCCTCAT \\
\hline & & & TGCAG & CAAT \\
\hline 6 & AAAAAGGTCTCC & GAAAGCTCCTTCCAC & GGAGCAAAATG & TTTTTGGATCCTCAG \\
\hline & CATGGGAATGCC & TGATCCTGTATACTA & TATAAAGGGAG & TGGTGGTGGTGGTG \\
\hline & CACCACCATCGA & GAATCTCССТTТАТА & ATTCTAGTATAC & GTGCTCGAGAAGTT \\
\hline & $\mathrm{GC}$ & CATTTTGCTCC & AGGATCAGTGG & CTTTTGCCGCCTCAT \\
\hline & & & AAGGAGCTTTC & CAAT \\
\hline 7 & AAAAAGGTCTCC & CGTGGGAAAGCTCC & TAAAGGGAGATT & TTTTTGGATCCTCAG \\
\hline & CATGGGAATGCC & TTCCATCGTTTCTGT & CTAGTATACAGA & TGGTGGTGGTGGTG \\
\hline & CACCACCATCGA & ATACTAGAATCTCCC & AACGATGGAAG & GTGCTCGAGAAGTT \\
\hline & $\mathrm{GC}$ & TTTA & GAGCTTTCCCAC & CTTTTGCCGCCTCAT \\
\hline & & & $\mathrm{G}$ & CAAT \\
\hline 8 & AAAAAGGTCTCC & GTCTTTCCTCTTGTC & САССТТСТСТСТ & TTTTTGGATCCTCAG \\
\hline & CATGGGAATGCC & CATCAGCAAAAGAC & GGTAGACACTTG & TGGTGGTGGTGGTG \\
\hline & CACCACCATCGA & AAGTGTCTACCAGA & TCTTTTGCTGAT & GTGCTCGAGAAGTT \\
\hline & $\mathrm{GC}$ & GAGAAGGTG & GGACAAGAGGA & CTTTTGCCGCCTCAT \\
\hline & & & AAGAC & CAAT \\
\hline
\end{tabular}




\begin{tabular}{l|llll} 
TCPTP $_{281}$ & AAAAAGGTCTCC & TTTTTGGATCCTCAA & N/A & N/A \\
& CATGGGAATGCC & TGATGATGATGATG & & \\
CACCACCATCGA & ATGCTCGAGTATAC & & \\
GC & ATTTTGCTCCTTCTA & & NTATAGCCATG \\
& & & \\
$T_{C P T P_{317}}$ & AAAAAGGTCTCC & TTTTTGGATCCTCAA & N/A \\
& CATGGGAATGCC & TGATGATGATGATG & & \\
& CACCACCATCGA & ATGCTCGAGTCTGTT & & \\
& GC & CCCATTGTATTTTC & & \\
& & AGTCATTATTTG & &
\end{tabular}


Table S3. Primers used for Gibson assembly.

\begin{tabular}{|c|c|c|}
\hline \# & F Primer & R Primer \\
\hline$P c \# 1-T C P T P_{V 9}-L O V 2$ & $\begin{array}{l}\text { AACAGAAATCGAAACAGATACA } \\
\text { GAGATGTAAGCCCATATGATCAC }\end{array}$ & $\begin{array}{l}\text { CACCACTATTGATATTTTCTAATTGT } \\
\text { AGTAGATGTACTGTATAATACGACTT } \\
\text { CACATC }\end{array}$ \\
\hline Pc\#2- TCPTP ${ }_{V 9}-L O V 2$ & $\begin{array}{l}\text { AATTAGAAAATATCAATAGTGGT } \\
\text { GAAACCAGAAC }\end{array}$ & $\begin{array}{l}\text { ATCTGTTTCGATTTCTGTTTTCTGGA } \\
\text { AAC }\end{array}$ \\
\hline$P c \# 1$ TCPTP ${ }_{K 287 A}$ & $\begin{array}{l}\text { CTCAGGTCGCAGACGTTTTGC } \\
\text { AG }\end{array}$ & $\begin{array}{l}\text { CCATCGGGCCTGTATACTAGAAT } \\
\text { CTCCCTTTATACATTTTGCTC }\end{array}$ \\
\hline Рс\#2 ТСРТР & $\begin{array}{l}\text { ATACAGGCCCGATGGAAAGA } \\
\text { ACTTTCTAAGGAAGACTTATC }\end{array}$ & $\begin{array}{l}\text { GTCTGCGACCTGAGCAACAACAT } \\
\text { G }\end{array}$ \\
\hline$P c \# 1$ TCPTP $V 9-D 290 A$ & $\begin{array}{l}\text { CTCAGGTCGCAGACGTTTTGC } \\
\text { AG }\end{array}$ & $\begin{array}{l}\text { CACTGGGCCTGCACGGAAGAGTC } \\
\text { CCC }\end{array}$ \\
\hline Pc\#2 TCPTPV9-D290A & $\begin{array}{l}\text { TGCAGGCCCAGTGGAAGGAG } \\
\text { CTTTCCCAC }\end{array}$ & $\begin{array}{l}\text { GTCTGCGACCTGAGCAACAACAT } \\
\text { G }\end{array}$ \\
\hline
\end{tabular}


Table S4. Primers used for Site-directed mutagenesis.

\begin{tabular}{c|ll}
$\#$ & \multicolumn{1}{c}{$\boldsymbol{F}$ Primer } & \multicolumn{1}{c}{$\boldsymbol{R}$ Primer } \\
\hline PTP1B $1286 A$ & GCCAAATTCATCATGGG & AAGCTCCTTCCACTGATCCTGCA \\
& GGACTCTGCCGTGCAGG & CGGCAGAGTCCCCCATGATGAA \\
& ATCAGTGGAAGGAGCTT & TTTGGC \\
& ATCATGGGGGACTCTTC & CTCGTGGGAAAGCTCCTTCCACT \\
& CGTGCAGGCTCAGTGGA & GAGCCTGCACGGAAGAGTCCCC \\
& AGGAGCTTTCCCACGAG & CATGAT \\
PTP1B $1 B_{E 297 A} 293 A$ & TCTTCCGTGCAGGATCA & GGGCTCCAGGTCCTCGTGGGAA \\
& GTGGAAGGCGCTTTCCC & AGCGCCTTCCACTGATCCTGCAC \\
& ACGAGGACCTGGAGCCC & GGAAGA \\
& GATCAGTGGAAGGAGCT & ATGCTCGGGTGGGGGCTCCAGG \\
& TTCCACGCGGACCTGG & TCCGCGTGGGAAAGCTCCTTCC \\
& AGCCCCACCCGAGCAT & ACTGATC
\end{tabular}


Table S5. Estimates of kinetic parameters.

\begin{tabular}{|c|c|c|c|c|c|}
\hline Construct & Substrate & kcat $(1 / \mathrm{s})$ & $K m(\mu M)$ & IC50 $(\mu M)$ & Figure \\
\hline$T C P T P_{V 9-D a r k}$ & $\mathrm{pNPP}$ & $6.0 \pm 0.4$ & $286 \pm 11$ & N/A & $2 \mathrm{D}$ \\
\hline$T_{C P T P}{ }_{\text {V-Light }}$ & pNPP & $3.1 \pm 0.3$ & $250 \pm 14$ & $\mathrm{~N} / \mathrm{A}$ & $2 \mathrm{D}$ \\
\hline PTPIB & $\mathrm{pNPP}$ & $2.1 \pm 0.2$ & $602 \pm 16$ & N/A & $3 \mathrm{D}$ \\
\hline$P T P 1 B_{S 286 A}$ & pNPP & $2.7 \pm 0.5$ & $1522 \pm 110$ & N/A & $3 \mathrm{D}$ \\
\hline$P T P 1 B_{D 289 A}$ & pNPP & $4.8 \pm 0.4$ & $958 \pm 52$ & N/A & $3 \mathrm{D}$ \\
\hline$P T P 1 B_{E 293 A}$ & $\mathrm{pNPP}$ & $3.4 \pm 0.4$ & $190 \pm 110$ & N/A & $3 \mathrm{D}$ \\
\hline$P T P 1 B_{E 297 A}$ & pNPP & $1.8 \pm 0.5$ & $602 \pm 16$ & N/A & $3 \mathrm{D}$ \\
\hline ТСРТP & $\mathrm{pNPP}$ & $2.0 \pm 0.1$ & $433 \pm 22$ & N/A & $3 \mathrm{~F}$ \\
\hline$T_{C P T P}{ }_{K 287 A}$ & pNPP & $2.1 \pm 0.3$ & $459 \pm 27$ & N/A & $3 F$ \\
\hline$T C P T P_{V 9}$ & pNPP & $14 \pm 1$ & $478 \pm 8.9$ & N/A & $3 G$ \\
\hline$T_{C P T P_{V 9-D 290 A}}$ & $\mathrm{pNPP}$ & $28 \pm 2$ & $750 \pm 6.5$ & N/A & $3 G$ \\
\hline$T_{C P T P} 317$ & pNPP & $3.2 \pm 0.5$ & $237 \pm 21$ & N/A & $\mathrm{S} 1$ \\
\hline$T_{C P T P} 281$ & pNPP & $1.3 \pm 0.2$ & $273 \pm 16$ & N/A & S1 \\
\hline ТСРTP & pNPP & $2.3 \pm 0.3$ & $394 \pm 45$ & N/A & S3A \\
\hline ТСРТP & 4-MUP & $5.6 \pm 0.4$ & $28.6 \pm 0.84$ & $\mathrm{~N} / \mathrm{A}$ & S3B \\
\hline ТСРТР & E-PEP & $11 \pm 2$ & $62.1 \pm 10$ & $\mathrm{~N} / \mathrm{A}$ & $\mathrm{S} 3 \mathrm{C}$ \\
\hline ТСРТР & P-PEP & $5.3 \pm 0.5$ & $23.5 \pm 5.0$ & N/A & S3D \\
\hline$T C P T P_{V 9}$ & pNPP & $4.7 \pm 0.4$ & $414 \pm 48$ & N/A & S3A \\
\hline$T C P T P_{V 9}$ & 4-MUP & $9.9 \pm 0.6$ & $25.9 \pm 1.4$ & N/A & S3B \\
\hline$T_{C P T P}^{V 9}$ & E-PEP & $24 \pm 4$ & $70.4 \pm 15$ & N/A & $\mathrm{S} 3 \mathrm{C}$ \\
\hline$T C P T P_{V 9}$ & P-PEP & $13 \pm 1$ & $28.9 \pm 6.6$ & N/A & S3D \\
\hline$P T P 1 B$ & pNPP & $4.8 \pm 0.5$ & $535 \pm 85$ & $0.90 \pm 0.2$ & S4A \\
\hline$T C P T P_{V 9}$ & pNPP & $5.8 \pm 0.9$ & $1380 \pm 100$ & $3.1 \pm 0.6$ & S4B \\
\hline ТСРТР & pNPP & $4.0 \pm 0.3$ & $464 \pm 21$ & $10 \pm 2$ & $\mathrm{~S} 4 \mathrm{C}$ \\
\hline
\end{tabular}

Error denotes standard error ( $\mathrm{n}=3$ independent reactions). 
Table S6A. Comparison of kinetic models for the inhibition of TCPTPvg by BBR.

\begin{tabular}{|c|c|c|c|c|c|}
\hline Model & $\begin{array}{c}S S E \\
\left(\mu M^{2} / s^{2}\right)\end{array}$ & $D F$ & Criteria & Reference & Fit Param. $(\mu M)$ \\
\hline Competitive & 0.0026 & 17 & $\Delta_{\mathrm{i}}=11.8$ & Noncompetitive & $\mathrm{Ki}=0.417$ \\
\hline Uncompetitive & 0.0021 & 17 & $\Delta_{\mathrm{i}}=7.3$ & Noncompetitive & $\mathrm{Ki}=2.27$ \\
\hline Noncompetitive & 0.0014 & 17 & & & $\mathrm{Ki}=3.06$ \\
\hline Mixed & 0.0013 & 16 & $\mathrm{p}=0.425$ & Noncompetitive & $\begin{array}{l}\mathrm{Kic}=1.96 \\
\mathrm{Kiu}=3.56\end{array}$ \\
\hline
\end{tabular}

Blue highlights indicate models of best fit.

Table S6B. Comparison of kinetic models for the inhibition of TCPTP by BBR.

\begin{tabular}{|c|c|c|c|c|c|}
\hline Model & $\begin{array}{c}S S E \\
\left(\mu M^{2} / s^{2}\right)\end{array}$ & $\overline{D F}$ & Criteria & Reference & Fit Param. $(\mu M)$ \\
\hline Competitive & 0.0034 & 17 & $\Delta_{\mathrm{i}}=13.6$ & Noncompetitive & $\mathrm{Ki}=0.796$ \\
\hline Uncompetitive & 0.0022 & 17 & $\Delta_{\mathrm{i}}=5.59$ & Noncompetitive & $\mathrm{Ki}=8.63$ \\
\hline Noncompetitive & 0.0016 & 17 & & & $\mathrm{Ki}=9.94$ \\
\hline Mixed & 0.0013 & 16 & $\begin{array}{c}p= \\
0.0707\end{array}$ & Noncompetitive & $\begin{array}{l}\mathrm{Kic}=3.30 \\
\mathrm{Kiu}=13.3\end{array}$ \\
\hline
\end{tabular}

Blue highlights indicate models of best fit.

Table S6C. Comparison of kinetic models for the inhibition of PTP1B by BBR.

\begin{tabular}{|c|c|c|c|c|c|}
\hline Model & $\begin{array}{c}S S E \\
\left(\mu M^{2} / s^{2}\right)\end{array}$ & $D F$ & Criteria & Reference & Fit Param. $(\mu M)$ \\
\hline Competitive & 0.0012 & 17 & $\Delta_{\mathrm{i}}=5.32$ & Noncompetitive & $\mathrm{Ki}=0.047$ \\
\hline Uncompetitive & 0.0015 & 17 & $\Delta_{\mathrm{i}}=9.41$ & Noncompetitive & $\mathrm{Ki}=0.720$ \\
\hline Noncompetitive & 0.00087 & 17 & & & $\mathrm{Ki}=0.900$ \\
\hline Mixed & 0.00052 & 16 & $\begin{array}{c}\mathrm{p}= \\
0.0316\end{array}$ & Noncompetitive & $\begin{aligned} \mathrm{Kic} & =0.187 \\
\mathrm{Kiu} & =1.44\end{aligned}$ \\
\hline
\end{tabular}

Blue highlights indicate models of best fit. 
Table S7. Crystallographic data.

\begin{tabular}{|c|c|}
\hline & $\begin{array}{c}\mathrm{PTP}_{1 B_{1-321}(\mathrm{D} 289 \mathrm{~A})} \\
\text { 7KEN }\end{array}$ \\
\hline \multicolumn{2}{|l|}{ Data collection } \\
\hline Space group & P $3{ }_{1} 21$ \\
\hline Number of crystals & 1 \\
\hline \multicolumn{2}{|l|}{ Cell dimensions } \\
\hline$a, b, c(\AA)$ & $88.34,88.34,104.19$ \\
\hline$\alpha, \beta, \gamma\left({ }^{\circ}\right)$ & $90.00,90.00,120.00$ \\
\hline Resolution $(\AA)$ & $76.48-1.80(1.83-1.80)^{*}$ \\
\hline$R_{\text {merge }}$ & $0.178(3.165)$ \\
\hline$I / \sigma I$ & $6.2(0.3)$ \\
\hline Completeness (\%) & $100.0(100.0)$ \\
\hline Redundancy & $10.8(10.9)$ \\
\hline \multicolumn{2}{|l|}{ Refinement } \\
\hline Resolution $(\AA)$ & $76.48-1.80(1.847-1.80)$ \\
\hline No. reflections & 40989 \\
\hline$R_{\text {work }} / R_{\text {free }}$ & $0.20 / 0.24$ \\
\hline \multicolumn{2}{|l|}{ No. atoms } \\
\hline Protein & 2633 \\
\hline Ligand/ion & 9 \\
\hline Water & 187 \\
\hline$B$-factor (all atoms) & 30.4 \\
\hline \multicolumn{2}{|c|}{ R.m.s. deviations (protein) } \\
\hline Bond lengths ( $\AA$ ) & 0.60 \\
\hline Bond angles $\left(^{\circ}\right)$ & 0.77 \\
\hline
\end{tabular}

*Values in parentheses correspond to the highest-resolution shell. 